\title{
The Action Functional in Non-Commutative Geometry
}

\author{
A. Connes \\ I.H.E.S., F-91440 Bures-sur-Yvette, France
}

\begin{abstract}
We establish the equality between the restriction of the Adler-ManinWodzicki residue or non-commutative residue to pseudodifferential operators of order $-n$ on an $n$-dimensional compact manifold $M$, with the trace which J. Dixmier constructed on the Macaev ideal. We then use the latter trace to recover the Yang Mills interaction in the context of non-commutative differential geometry.
\end{abstract}

\section{Introduction}

The non-commutative residue was discovered in the special case of one dimensional symbols by Adler [1] and Manin [8] in the context of completely integrable systems. In a quite remarkable work [13], Wodzicki proved that it could still be defined in arbitrary dimension and gave the only non-trivial trace, noted Res, for the algebra of pseudodifferential operators of arbitrary order. Given such an operator $P$ on the manifold $M, \operatorname{Res} P$ is the coefficient of $\log t$ in the asymptotic expansion of $\operatorname{Trace}\left(P e^{-t \Delta}\right)$, where $\Delta$ is a Laplacian. Equivalently it is the residue at $s=0$ of the $\zeta$ function $\zeta(s)=\operatorname{Trace}\left(P \Delta^{-s}\right)$. It is not the usual regularisation $\zeta(0)$ of the trace, and it vanishes on any $P$ of order strictly less than $-\operatorname{dim} M$, and on any differential operator. In general this trace: Res, has no positivity property, i.e. one does not have $\operatorname{Res}\left(P^{*} P\right) \geqq 0$. However its restriction to operators of order $-n, n=\operatorname{dim} M$ is positive. This restriction of Res to pseudodifferential operators of order $-n$ was discovered and studied by Guillemin [14]. Even though it is easier to handle than the general residue, it will be of great help for our purpose which is to show how conformal geometry fits with [3], the case of Riemannian geometry being treated in [5].

Our first result is the equality between Res and a trace on the dual Macaev ideal, introduced by Dixmier in [6] in order to show that the von Neumann algebra $\mathscr{L}(\mathscr{H})$ of all bounded operators in Hilbert space possessed non-trivial tracial weights. I am grateful to J. Dixmier for explaining his result to me and to $D$. Voiculescu for helpful conversations on the subject of Macaev ideals. Thus we recall that, given a Hilbert space $\mathscr{H}$, the Macaev ideal $\mathscr{L}^{\omega}(\mathscr{H})$ is the ideal of 
compact operators $T$, whose characteristic values satisfy: [7]

$$
\sum_{1}^{\infty} \frac{1}{n} \mu_{n}(T)<\infty
$$

It contains all the Schatten classes $\mathscr{L}^{p}(\mathscr{H})$ for finite $p$, and the dual ideal, which we denote $\mathscr{L}^{1+}$ consists of all compact operators $T$, whose characteristic values satisfy:

$$
\operatorname{Sup}_{N>1} \frac{1}{\log N} \sum_{1}^{N} \mu_{n}(T)<\infty .
$$

Gifted with the obvious norm it is a non-separable Banach space containing strictly the ideal $\mathscr{L}^{1}$ as well as the closure of finite rank operators (thus $\mathscr{L}^{1}$ is not norm dense in $\mathscr{L}^{1+}$ for the natural norm of the latter).

Now in [6], J. Dixmier showed that for any mean $\omega$ on the amenable group of upper triangular two by two matrices, one gets a trace on $\mathscr{L}^{1+}$, given by the formula:

$$
\operatorname{Tr}_{\omega}(T)=\lim _{\omega} \frac{1}{\log N} \sum_{1}^{N} \lambda_{n}(T)
$$

when $T$ is a positive operator, $T \in \mathscr{L}^{1+}$, with eigenvalues $\lambda_{n}(T)$ in decreasing order, and $\lim _{\omega}$ is the linear form on bounded sequences defined in [6] using $\omega$.

We shall prove in Sect. 1 that when $T$ is pseudodifferential of order $-\operatorname{dim}(M)$, the value of $\operatorname{Tr}_{\omega}(T)$ does not depend upon $\omega$ and is equal to $\operatorname{Res}(T)$. In Sect. 2 we shall apply the above result to show how one can deduce ordinary differential forms and the natural conformal invariant norm on them from the quantized forms which we introduced in [3]. The key point is that we do not need to take a "classical limit" to achieve this goal but only to use the Dixmier trace appropriately. In particular we obtain a simple formula for the conformal structure in terms of the operator $F, F^{2}=1$, given by the polar decomposition of the Dirac operator.

In Sects. 3 and 4 after discussing the analogue of the Yang Mills action in the context of non-commutative differential geometry and showing, as expected, that 4 is the critical dimension, we exploit the above construction to show that if $d=4$ the leading divergency of the action is the usual local Yang Mills action. The latter result was announced on several occasions.

\section{The Main Equality}

Theorem 1. Let $M$ be a compact $n$-dimensional manifold, E a complex vector bundle on $M$, and $P$ a pseudodifferential operator of order - $n$ acting on sections of $E$. Then the corresponding operator $P$ in $\mathscr{H}=L^{2}(M, E)$ belongs to the Macaev ideal $\mathscr{L}^{1+}(\mathscr{H})$ and one has:

$$
\operatorname{Trace}_{\omega}(P)=\frac{1}{n} \operatorname{Res}(P)
$$

for any invariant mean $\omega$.

Note first that both $\mathscr{L}^{1+}(\mathscr{H})$ and Trace ${ }_{\omega}$ are invariant under similarities $T . T^{-1}$ with $T$ and $T^{-1}$ bounded, so that the choice of inner product in the space of $L^{2}$ sections of $E$ is irrelevant. 
Proof. Since $\mathscr{L}^{1+}(\mathscr{H})$ contains $\mathscr{L}^{1}(\mathscr{H})$, and any element of the latter is in the kernel of $\operatorname{Tr}_{\omega}$, it follows that we can neglect smoothing operators and we just need to prove the statements locally. Thus to show that $P \in \mathscr{L}^{1+}(\mathscr{H})$ we may assume that $M$ is the standard $n$ torus $\mathbb{T}^{n}$ and $E$ the trivial line bundle. Then $P=T(1+\Delta)^{-n / 2}$, where $T$ is bounded and $\Delta$ is the Laplacian of the (flat) torus. Thus as $\mathscr{L}^{1+}$ is an ideal it is enough to check that $(1+\Delta)^{-n / 2} \in \mathscr{L}^{1+}$, which is obvious. In fact the characteristic values of $(1+\Delta)^{-n / 2}$ are the $\left(1+l^{2}\right)^{-n / 2}$, where the $l$ 's are the lengths of elements in the lattice $\Gamma=\mathbb{Z}^{n}$. Thus we see that the limit of $\frac{1}{\log N} \sum_{1}^{N} \lambda_{j}$ when $N$
goes to $\infty$, does exist for this operator so that, for any $\omega:$

$$
\operatorname{Trace}_{\omega}\left((1+\Delta)^{-n / 2}\right)=\frac{1}{n} \int_{S^{n-1}} d \sigma=\frac{1}{n} 2 \pi^{\frac{n-1}{2}} / \Gamma\left(\frac{n-1}{2}\right) .
$$

Let us now prove the main equality. We may assume that $M$ is the standard $n$-sphere $S^{n}$. Since Trace $_{\omega}$ is positive and vanishes on $\mathscr{L}^{1}(\mathscr{H})$ it defines a positive linear form on symbols of order $-n$, because it only depends upon the principal symbol $\sigma_{-n}(P)$ for $P$ of order $-n$. Since a positive distribution is a measure, we get a measure on the unit sphere cotangent bundle of $S^{n}$. But as $\operatorname{Tr}_{\omega}$ is a trace, the latter measure is invariant under the action of any isometry of $S^{n}$, and hence is proportional to the volume form on $\left(T^{*} S^{n}\right)_{1}=\left\{(x, \xi) \in T^{*} S^{n} ;\|\xi\|=1\right\}$. By the above computation the constant of proportionality is $\frac{1}{n}(2 \pi)^{-n}$, thus:

$$
\operatorname{Trace}_{\omega}(P)=\frac{1}{n}(2 \pi)^{-n} \int_{\left(T^{*} S^{n}\right)_{1}} \sigma_{-n}(P) d v
$$

for any $P$ of order $-n$ and any $\omega$. As the right-hand side is the formula for $\frac{1}{n} \operatorname{Res}(P)$, we get the conclusion.

Corollary 2. All the traces $\operatorname{Tr}_{\omega}$ agree on pseudodifferential operators of order $-\operatorname{dim} M$, on a manifold $M$. One can then conclude that suitable averages of the sequence $\frac{1}{\log N} \sum_{1}^{N} \lambda_{j}(P)$ do
converge, when $N \rightarrow \infty$, to this common value.

\section{Conformal Geometry}

Let $M$ be a compact Riemannian manifold of dimension $n$, and $A^{1}=C^{\infty}\left(M, T^{*} M\right)$ be the space of smooth 1 -forms on $M$. There is a natural norm on $A^{1}$ which depends only upon the conformal structure of $M$. If $\operatorname{dim} M=2$, it is the ordinary Dirichlet integral: $\int\|\omega\|^{2} d v=\int \omega \wedge * \omega$. If $\operatorname{dim} M=n$, it is the $L^{n}$ norm, given by the $\left(n^{\text {th }}\right.$ root of) following integral:

$$
(\|\omega\|)^{n}=\int\|\omega(x)\|^{n} d^{n} x .
$$

In [3] we introduced (assuming that $M$ is $\operatorname{Spin}^{c}$ ) the quantized differential forms on $M$, obtained as operators of the form $\sum a d b ; a, b \in C^{\infty}(M)$, in the Hilbert space $\mathscr{H}$ of $L^{2}$ spinors on $M$. Here $d b$ is given by the commutator $i[F, b]$, where the operator $F, F^{2}=1$, is the sign $D|D|^{-1}$ of the Dirac operator. (We can ignore the non-invertibility of $D$, since it only modifies $F$ by a finite rank operator.) 
The next result shows how to pass from quantized 1-forms to ordinary forms, not by a classical limit, but by a direct application of the Dixmier trace.

Theorem 3. Let $M$ be a Spin ${ }^{c}$ Riemannian manifold of dimension $n>1$, $\mathscr{H}=L^{2}(M, S)$ the Hilbert space of $L^{2}$ spinors, $F=D|D|^{-1}$ the sign of the Dirac operator. Let $\mathscr{A}=C^{\infty}(M)$ be the algebra of smooth functions on $M$ and $\Omega^{1}=\{\Sigma a[F, b] ; a, b \in \mathscr{A}\}$ be the $\mathscr{A}$-bimodule of quantized forms of degree 1.

1) For any $\alpha \in \Omega^{1}$ one has $|\alpha|^{n} \in \mathscr{L}^{1+}(\mathscr{H})$.

2) There exists a unique bimodule linear map $\Omega^{1} \stackrel{c}{\longrightarrow} A^{1}$ such that $c(i[F, a])=d a \forall a \in C^{\infty}(M)$. This map is surjective and the image of the self adjoint elements of $\Omega^{1}$ are the real forms.

3) For any $\alpha=\alpha^{*} \in \Omega^{1}$ one has $\operatorname{Trace}_{\omega}\left(|\alpha|^{n}\right)=\lambda_{n} \int\|c(\alpha)\|^{n}$ with $\lambda_{n}=2(2 \pi)^{-n / 2} \Gamma\left(n-\frac{1}{2}\right) \Gamma\left(\frac{n-1}{2}\right)^{-1} \Gamma(n+1)^{-1}$.

Proof. 1. By construction $\alpha$ is a pseudodifferential operator of order -1 , so that $|\alpha|^{n}$ is also a pseudodifferential operator and is of order $-n$. The conclusion follows from Theorem 1.

2. For $x \in M$ let $C_{x}=\operatorname{Cliff}_{\mathbb{C}}\left(T_{x}^{*} M\right)$ be the complexified Clifford algebra of the cotangent space $T_{x}^{*} M$ of $M$ at $x$. One has $C_{x}=\operatorname{End}\left(S_{x}\right)$, where $S$ is the Spinor bundle. For each $\xi \in T_{x}^{*} M$ we let $\gamma(\xi) \in C_{x}$ be the corresponding $\gamma$ matrix, $\gamma(\xi)=\gamma(\xi)^{*}, \gamma(\xi)^{2}=\|\xi\|^{2}$, and we extend $\gamma$ to a linear map of $T_{x, \mathbb{C}}^{*}(M)$ to $C_{x}$. Given $a \in \mathscr{A}=C^{\infty}(M)$, the symbol of order -1 of $[F, a]$ is the Poisson bracket $\{\sigma, a\}$, where $\sigma(x, \xi)=\gamma(\xi) /\|\xi\|$, and thus its restriction to the unit sphere is the transverse part $\varrho(x, \xi)=\gamma(d a-<d a, \xi>\xi)$ of $\gamma(d a)$. It is a homogeneous function of degree -1 on $T_{x}^{*} M$ with values in $C_{x}$. Now provided $n>1$, a vector $\eta \in T_{x}^{*}$ is uniquely determined by the transverse part $\xi \rightarrow \eta-<\eta, \xi>\xi$, as a function of $\xi \in S_{x}^{*}$, and this

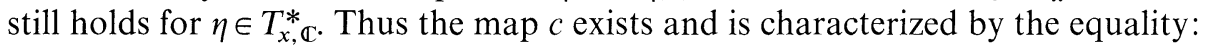

$$
\sigma_{-1}(\alpha)(x, \xi)=\gamma(c(\alpha)(x)-<c(\alpha)(x), \xi>\xi) \forall(x, \xi) \in S^{*} M .
$$

The image of $\sum a i[F, b] \in \Omega^{1}$ is $\sum a d b \in A^{1}$ so the surjectivity of $c$ is clear. The image of $a i[F, b]+(a i[F, b])^{*}$ is $a d b+\left(d b^{*}\right) a^{*}$ which is a real form, so 2 . follows.

3. The absolute value of $\gamma(\eta)$ for $\eta \in T_{x}^{*}(M)$ (but not its complexification) is $\|\eta\| 1$, where 1 is the unit of $C_{x}$. Thus by Theorem 1 we have:

$$
\text { Trace } \left._{\omega}(|\alpha|)^{n}\right)=\frac{(2 \pi)^{-n}}{n} \int_{S^{*} M}\left\|\alpha_{x}-<\alpha_{x}, \xi>\xi\right\|^{n} \operatorname{trace}(1) d^{n} x d^{n-1} \xi .
$$

Here trace $(1)=\operatorname{dim}\left(S_{x}\right)=2^{n / 2}$. Thus we just need to show that for any $\eta \in \mathbb{R}^{n}$ one has $\int_{S^{n-1}}\|\eta-<\eta, \xi>\xi\|^{n}\left(d^{n-1} \xi\right)=2^{-n / 2} \lambda_{n}\|\eta\|^{n}$. By homogeneity and invariance under rotations we are reduced to the computation of an integral, which is obviously $>0$ for $n>1$.

As an immediate corollary of the theorem we see that the Fredholm module $(\mathscr{H}, F)$ allows us to recover both the bimodule of 1 -forms $A^{1}$ with the ordinary differentiation: $\mathscr{A} \stackrel{d}{\longrightarrow} A^{1}$ (given by $a \rightarrow$ Class of $i[F, a]$ ), and also the conformal structure of $M$ since the $L^{n}$ norm on $A^{1}$ uniquely determines it. 
Another equivalent way to formulate the result is to consider for each $n$ the ideal $\mathscr{L}^{n+}, n^{\text {th }}$ root of $\mathscr{L}^{1+}$, in $\mathscr{L}(\mathscr{H})$,

$$
\mathscr{L}^{n+}=\left\{T \in \mathscr{L}(\mathscr{H}), T \text { compact, } \operatorname{Sup}_{N}\left(\frac{1}{\log N} \sum_{1}^{N} \mu_{j}(T)^{n}\right)<\infty\right\},
$$

and the ideal $\mathscr{L}_{0}^{n+}$ which is the norm closure, for the norm of $\mathscr{L}^{n+}$, of operators of finite rank (cf. [7]). Then on an $n$-dimensional manifold $M$ as above the quantized 1 -forms are all in $\mathscr{L}^{n+}$, and the ordinary forms are obtained by moding out $\mathscr{L}_{0}^{n+}$ $\subset \mathscr{L}^{n+}$. The ordinary differential is obtained in the same way from the quantized differential $a \rightarrow i[F, a] \in \Omega^{1}$.

For forms of arbitrary degree there are two more points which we have to clarify before we can handle the Yang Mills action. Given an $n$-dimensional Euclidean space $E$, we let $\Pi_{E}$ be the homomorphism of the tensor algebra $T(E)$ in $C^{\infty}\left(S_{E}\right.$, Cliff $(E)$ ), (the algebra of smooth maps from the unit sphere $S_{E}=\{\xi \in E$, $\|\xi\|=1\}$ to the Clifford algebra of $E$ ) obtained from the linear map $\eta \rightarrow \varrho(\eta)$, $\varrho(\eta)(\xi)=\gamma(\eta-<\eta, \xi>\xi) \forall \xi \in S_{E}$.

We let $J(E)$ be the kernel of $\Pi_{E}$.

Lemma 4. With the notations of Theorem 3 , let $\Omega^{k}$ be the the $\mathscr{A}$-bimodule of quantized forms of degree $k$.

1. For $1 \leqq k \leqq n$ one has $\Omega^{k} \subset \mathscr{L}^{n / k+}(\mathscr{H})$ and the direct sum $\bigoplus_{n}^{n} \Omega_{0}^{k}$, with $\Omega_{0}^{k}=\mathscr{L}^{n / k+} \cap \Omega^{k}$ is a two sided ideal in the algebra $\bigoplus_{0}^{n} \Omega^{k}=\Omega^{*}$.

2. The principal symbol map gives a canonical isomorphism c of graded algebras, from $\Omega^{*} / \Omega_{0}^{*}$ to the graded algebra of smooth sections of the vector bundle $\oplus_{0}^{n} E_{k}$, where $E_{k}$ is obtained from the cotangent bundle by applying the functor:

$$
E \rightarrow T^{k}(E) / J(E) \cap T^{k}(E)=f_{k}(E) .
$$

Proof. 1. Any element of $\Omega^{k}$ is a pseudodifferential operator $P$ of order $-k$; thus $|P|^{n / k}$ is of order $-n$ and Theorem 1 applies. The Holder inequality also holds for the ideals $\mathscr{L}^{p+}$ and shows that $\mathscr{L}^{p_{1}+} \times \mathscr{L}^{p_{2}+} \subset \mathscr{L}^{p_{3}+}, \frac{1}{p_{3}}=\frac{1}{p_{1}}+\frac{1}{p_{2}}$ and also that $\mathscr{L}_{0}^{p_{1}+} \times \mathscr{L}^{p_{2}+} \subset \mathscr{L}_{0}^{p_{3}+}, \mathscr{L}^{p_{1}+} \times \mathscr{L}_{0}^{p_{2}+} \subset \mathscr{L}_{0}^{p_{3}+}$ (cf. [7]).

2. First, by Theorem 1 , an element $P$ of $\Omega^{k}$ belongs to $\mathscr{L}_{0}^{n / k+}$ if and only if its principal symbol vanishes. (If it does then the operator is of order $<-k$ and hence even belongs to $\mathscr{L}^{n / k}$; if it does not then the Dixmier trace of $|P|^{n / k}$ does not vanish.) The quotient $\Omega^{k} / \Omega_{0}^{k}$ is a commutative bimodule over $\mathscr{A}=C^{\infty}(M)$, and since any element of $\Omega^{k}$ is a finite sum of products of $k$ elements of $\Omega^{1}$, the symbols $\sigma_{-k}(P)$, $P \in \Omega^{k}$ are exactly the smooth sections of $f_{k}\left(T^{*} M\right)$.

For our purpose we only need to determine $f_{1}$ and $f_{2}$. For $n>1$ we have seen that $f_{1}(E)=E$. For $n>2$ let us show that $J(E) \cap T^{2}(E)=\{0\}$, i.e. that the map $\Pi_{E}$ is injective on tensors of rank 2. Since $J(E)$ is invariant under the action of the orthogonal group $O(E)$, it is enough to check that $\Pi_{E}$ is non-zero on the three irreducible subspaces of $T^{2}(E)$, namely a) antisymmetric tensors b) symmetric traceless tensors c) the inner product (viewed as a symmetric tensor). Since $n>2$ we 
can take $\eta_{1}, \eta_{2} \in E$ linearly independent, and $\xi$, $\|\xi\|=1$, orthogonal to both, to get that $\Pi_{E}\left(\eta_{1} \otimes \eta_{2}-\eta_{2} \otimes \eta_{1}\right) \neq 0$. The image by $\Pi_{E}$ of the symmetric tensor $\eta_{1} \otimes \eta_{2}$ $+\eta_{2} \otimes \eta_{1}\left(\eta_{i} \in E\right)$ is the scalar valued function on $S_{E}: \Pi_{E}\left(\eta_{1} \otimes \eta_{2}+\eta_{2} \otimes \eta_{1}\right)(\xi)$ $=\left\langle\eta_{1}, \eta_{2}\right\rangle-\left\langle\eta_{1}, \xi\right\rangle\left\langle\eta_{2}, \xi\right\rangle$. This is enough to show that $\Pi_{E}$ is non-zero and hence injective on tensors of type a) b) or c). Thus we get:

Lemma 5. If $\operatorname{dim} E>2, f_{2}(E)=T^{2}(E)$.

The next point that we need to clarify is that even though $f=\Omega^{*} / \Omega_{0}^{*}$ is a graded algebra of tensors on the manifold $M$, and $c$ is a homomorphism from the graded algebra $\Omega^{*}$ to $\Omega^{*} / \Omega_{0}^{*}$, we do not have a natural differential in $f$. The point is that the ideal $\Omega_{0}^{*}$ is not in general stable under the map:

$$
\alpha \in \Omega^{k} \rightarrow d \alpha=i\left(F \alpha-(-1)^{k} \alpha F\right) \in \Omega^{k+1} .
$$

However since $d^{2}=0$, this is easily cured:

Lemma 6. 1. The direct sum $\Omega_{00}^{*}=\bigoplus_{0}^{n} \Omega_{00}^{k}$ with $\Omega_{00}^{k}=\left\{\alpha \in \Omega_{0}^{k}, d \alpha \in \Omega_{0}^{k+1}\right\}$ is a graded differential two sided ideal in the graded differential algebra $\Omega^{*}$.

2. The map $\tilde{c}, \tilde{c}(\alpha)=(c(\alpha), c(d \alpha))$ is a linear injection of the quotient $\Omega^{k} / \Omega_{00}^{k}$ in the space of sections of the bundle $f_{k}\left(T^{*}\right) \oplus f_{k+1}\left(T^{*}\right)$.

Proof. 1. We just have to check that it is a two sided ideal, which follows from Lemma 4 1) and the equality $d\left(\alpha_{1} \alpha_{2}\right)=\left(d \alpha_{1}\right) \alpha_{2}+(-1)^{\partial_{1}} \alpha_{1} d \alpha_{2}$.

2. Apply Lemma 4 2).

Assuming $n>2$ let us determine the image $\tilde{c}\left(\Omega^{1}\right)$, i.e. the pairs $(c(\alpha), c(d \alpha))$ when $\alpha$ varies in $\Omega^{1}$.

Lemma 7. For $n>2, \tilde{c}\left(\Omega^{1}\right)$ consists of all smooth tensors $(\omega, \beta)$, where $\omega$ is of rank 1 , $\beta$ of rank 2 and one has:

$$
A \beta=d \omega,
$$

where $A$ is the projection on antisymmetric tensors of rank 2 .

Proof. It is enough to check the equation for the pair $\omega=c(\alpha), \beta=c(d \alpha)$ with $\alpha=a d b ; a, b \in C^{\infty}(M)$. Then by Theorem 32$), c(\alpha)$ is the 1 -form $a d b$ and since $d \alpha=d a d b$, we see that $A \beta$ is the antisymmetric tensor $\frac{1}{2}(d a \otimes d b-d b \otimes d a)$, thus the equality $A \beta=d \omega$. It remains to show that $\tilde{c}\left(\Omega^{1}\right)$ contains all the smooth symmetric tensors of rank 2. Now with $\alpha=a d b$ as above and $x \in C^{\infty}(M)$ we have $c(x \alpha-\alpha x)=0$ and $c(d(x \alpha-\alpha x))=c((d x) \alpha+\alpha(d x))$. Thus $\tilde{c}(x \alpha-\alpha x)$ is the smooth symmetric two tensor $(d x) \alpha+\alpha(d x)$. As every smooth symmetric two tensor is a finite sum of such terms we get the conclusion.

\section{The Action Functional in Non-Commutative Differential Geometry}

We begin this section by a very simple example, the case of the circle $S^{1}$, where we show that using our quantized differential forms, the quantized flat connections correspond exactly to the Grassmannian which plays a fundamental role in the theory of totally integrable systems [9]. 
Thus we let $\mathscr{A}=C^{\infty}\left(S^{1}\right)$ be the algebra of smooth functions on $S^{1}$ and let $(\mathscr{H}, F)$ be the Fredholm module over $\mathscr{A}$ given by $\mathscr{H}=L^{2}\left(S^{1}\right)$ and $F=2 P-1$, where $P$ is the Toeplitz projection. In other words the operator $F$ multiplies the $n^{\text {th }}$ Fourier component of $\xi \in L^{2}\left(S^{1}\right)$ by 1 if $n \geqq 0$ and -1 otherwise.

Lemma 8. The space $\Omega^{1}=\left\{\sum a[F, b] ; a, b \in \mathscr{A}\right\}$ of 1-forms is dense in the space $\mathscr{L}^{2}(\mathscr{H})$ of Hilbert Schmidt operators.

Proof. Let $u \in \mathscr{A}$ be the function $u(\theta)=\exp i \theta, \theta \in S^{1}$. The operator $\frac{1}{2} u^{-1}[F, u]$ is the rank one projection on the subspace $\mathbb{C} e_{0}$, where $\left(e_{n}\right)_{n \in \mathbb{Z}}$ is the canonical basis of $\mathscr{H}=L^{2}\left(S^{1}\right), \quad e_{n}(\theta)=\exp ($ in $\theta), \quad \forall \theta \in S^{1} . \quad$ Thus the quantized forms $\omega_{n, m}$ $=u^{n}\left(\frac{1}{2} u^{-1}[F, u]\right) u^{m}$ form the natural orthonormal basis of $\mathscr{L}^{2}(\mathscr{H})$.

We cannot entirely justify the choice of the Hilbert Schmidt norm in the above lemma, since it happens in dimension 1, that 1-forms are traceable. (As we saw above, by Theorem 1, it is not true that 1 -forms belong to $\mathscr{L}^{n}$ for an $n$-dimensional manifold, $n>1$.) The only sensible justification is that the definition of the character of the Fredholm module only requires that 1-forms be of Hilbert Schmidt class, and is continuous in this norm (cf. [3]). Next consider the trivial line bundle, with fiber $\mathbb{C}$, on $S^{1}$, or equivalently the finite projective module $\mathscr{E}=C^{\infty}\left(S^{1}\right)$ over $\mathscr{A}$. Then as in [3, Definition 18, p. 110] a connection $\nabla$ on $\mathscr{E}$ is given by a linear map $\nabla: \mathscr{E} \rightarrow \mathscr{E} \otimes_{\mathscr{A}} \Omega^{1}$ such that

$$
\nabla(\xi \cdot x)=(\nabla \xi) x+\xi \otimes d x,
$$

where here $d x=i[F, x]$, according to our definition of the quantized differential. We endow the above line bundle with its obvious metric, i.e. we view $\mathscr{E}$ as a $C^{*}$ module over $\mathscr{A}$, with $\langle\xi, \eta\rangle(\theta)=\bar{\xi}(\theta) \eta(\theta), \forall \theta \in S^{1}, \forall \xi, \eta \in \mathscr{E}$. Obviously a connection on $\mathscr{E}$ is specified by the 1 -form $\alpha=\nabla 1$, and the latter is an arbitrary element of $\Omega^{1}$. Moreover the connection associated to $\alpha$ is compatible with the metric (cf. [4]), (i.e. such that $\langle\nabla \xi, \eta\rangle+\langle\xi, \nabla \eta\rangle=d\langle\xi, \eta\rangle \forall \xi, \eta \in \mathscr{E}$ ) iff $\alpha+\alpha^{*}=0$.

We thus get the elementary but significant result:

Theorem 9. The map $\nabla \rightarrow \frac{1}{2}(1+F)-\frac{1}{2} i \nabla(1)$ is a one-to-one bijection from flat compatible and square integrable connections on $\mathscr{E}$ with the restricted Grassmannian. It is equivariant with respect to the natural action of $C^{\infty}\left(S^{1}, U(1)\right)$.

Proof. First $\nabla$ is characterized by $\alpha=\nabla(1)$ and is compatible iff $\alpha^{*}=-\alpha$, and square integrable iff $\alpha \in \mathscr{L}^{2}$; thus by Lemma 8 , without the flatness condition the allowed $\alpha$ 's are the skew adjoint elements of $\mathscr{L}^{2}(\mathscr{H})$. Now (cf. [9]) the restricted Grassmannian consists exactly of the idempotents $Q, Q=Q^{*}$ such that $Q-P \in \mathscr{L}^{2}$. Thus if we set $Q=\frac{1}{2}(1+F)-\frac{1}{2} i \alpha$, we just need to check that $Q^{2}=Q$ iff $\nabla_{\alpha}$ is flat, i.e. iff one has $i(F \alpha+\alpha F)+\alpha^{2}=0$, which is obvious. The unitary group $\mathscr{U}=C^{\infty}\left(S^{1}, U(1)\right)$ of End $\left.\mathbb{A}_{\mathscr{Q}} \mathscr{E}\right)$ acts by gauge transformations on compatible connections (cf. [4]) with $\gamma_{u}(\nabla)=u \nabla u^{-1}$ for $u \in \mathscr{U}$, or equivalently $\gamma_{u}(\alpha)=u i\left[F, u^{-1}\right]+u \alpha u^{-1}$. Thus the corresponding $Q_{\alpha}$ is replaced by $u Q_{\alpha} u^{-1}$.

A similar statement holds for the bundle with fiber $\mathbb{C}^{n}$, with $\mathscr{U}$ replaced by $C^{\infty}\left(S^{1}, U(n)\right)$.

In relation with [2] and [12] we also want to point out that on the space of all compatible connections (i.e. all $\alpha=-\alpha^{*}$ in $\mathscr{L}^{2}(\mathscr{H})$ ) one has a natural Chern- 
Simons action given by

$$
I(\alpha)=\int\left(\alpha d \alpha+\frac{2}{3} \alpha^{3}\right),
$$

where the integral is the trace and as usual $d \alpha$ is the graded commutator $d \alpha=i(F \alpha+\alpha F)$.

But let us now pass to the analogue of the Yang Mills action. The set up is, as in [3] and as above, fixed by $a_{*}$ algebra $\mathscr{A}$ and a Fredholm module $(\mathscr{H}, F)$ over $\mathscr{A}$ which is $p$-summable, i.e. $[F, x] \in \mathscr{L}^{p}(\mathscr{H})$ for some finite $p$, which as explained in [3] has to do with dimension. We are also given the analogue of a Hermitian bundle, i.e. a finite projective module $\mathscr{E}$ over $\mathscr{A}$, with an $\mathscr{A}$ valued inner product (cf. [4]). This latter data can be ignored for a first reading and specialized to $\mathscr{E}=\mathscr{A}$ with $\langle a, b\rangle=a^{*} b \in \mathscr{A}$.

Then using the differential algebra of quantized differential forms, $\Omega^{k}=\left\{\sum a^{0} d a^{1} \ldots d a^{k} ; a^{j} \in \mathscr{A}, d a=i[F, a]\right\}$ (cf. [3]) we get the notions of connection, compatible connection, curvature relative to $\mathscr{E}$. For $\mathscr{E}=\mathscr{A}$ a connection is just an element $\alpha$ of $\Omega^{1}$, it is compatible iff $\alpha^{*}=-\alpha$ and its curvature is $\theta=d \alpha+\alpha^{2}$ $=i(F \alpha+\alpha F)+\alpha^{2}$. (cf. [3, p. 110] and [4]). Using [3, Lemma 1, p. 56], we get:

Theorem 10. 1. The action $I_{+}(\alpha)=\|\theta\|_{H S}^{2}$ is finite if $p \leqq 4$.

2. When $p \leqq 4$, the action $I_{+}$is a quartic positive function of $\alpha$ invariant under the action of the gauge group of second kind

$$
\mathscr{U}=\left\{u \in \operatorname{End}(\mathscr{E}) ; u u^{*}=u^{*} u=1\right\} .
$$

Proof. For the sake of clarity we take $\mathscr{E}=\mathscr{A}$. By construction $\theta=d \alpha+\alpha^{2} \in \Omega^{2}$, and by [3, Lemma 1, p. 56] one has $\Omega^{k} \subset \mathscr{L}^{p / k}$, so that $\Omega^{2} \subset \mathscr{L}^{p / 2}$. Thus $\theta$ is Hilbert Schmidt when $p / 2 \leqq 2$, i.e. when $p \leqq 4$. If we replace $\alpha$ by $\gamma_{u}(\alpha)=u d u^{-1}+u \alpha u^{-1}$, the curvature $\theta$ is replaced by $u \theta u^{-1}$ so that the statement 2 . is obvious.

It is well known that the dimension $n=4$ is the relevant dimension for the classical Yang Mills action since it is only for $n=4$ that it is conformally invariant, but for the action $I_{+}$the situation is slightly different: 1 . The action $I_{+}$is finite only if the degree of summability $p$ is $\leqq 4,2$. For a 4-dimensional manifold $M$, the Fredholm module $(\mathscr{H}, F)$ on $C^{\infty}(M)$ given by Theorem 3 is $p$ summable for any $p=4+\varepsilon, \varepsilon>0$ but not for $p=4$. Thus in this case the action $I_{+}$is divergent. However by Lemma 4 one has $\Omega^{2} \subset \mathscr{L}^{2+}$ so that the divergence of $\|\theta\|_{H S}^{2}=\operatorname{Trace}\left(\theta^{*} \theta\right)$ is only $\operatorname{logarithmic}\left(\theta^{*} \theta \in \mathscr{L}^{1+}\right)$ and the principal term (i.e. the coefficient of $\log K$ in terms of a cut off $K$ ) is given by the Dixmier trace $\operatorname{Trace}_{\omega}\left(\theta^{*} \theta\right)$. In the next section we shall fully identify this leading term in $I_{+}$with the classical Yang Mills action.

\section{The Leading Term of the Action in 4 Dimensions}

Let $M$ be a 4 dimensional compact smooth Riemannian manifold. We assume that $M$ is $\operatorname{Spin}^{c}$ and let $(\mathscr{H}, F)$ be the Fredholm module over $\mathscr{A}=C^{\infty}(M)$, with $\mathscr{H}$ the Hilbert space of $L^{2}$ spinors and $F=D|D|^{-1}$, where $D$ is the Dirac operator. We let $\left(\Omega^{*}, d\right)$ be the graded differential algebra of quantized forms, and define as in Sect. 3 the notion of compatible connection for a Hermitian vector bundle $E$ over $M$. This 
involves the module $\mathscr{E}=C^{\infty}(M, E)$ (of sections of $E$ ) over $\mathscr{A}$ and the $\mathscr{A}$-valued inner product given by the metric of $E$. By construction (cf. [3]) the curvature $\theta$ is an element of $\operatorname{Hom}_{\mathscr{A}}\left(\mathscr{E}, \mathscr{E} \otimes \mathscr{\mathscr { A }}_{\mathscr{A}} \Omega^{2}\right)$, but since here $\Omega^{2}$ acts in the Hilbert space $\mathscr{H}$, we can view $\theta$ as an operator in the Hilbert space $\mathscr{E} \otimes \otimes_{\mathscr{A}} \mathscr{H}$. The inner product of the latter space is given by (cf. [4]) $\left\langle\xi \otimes \eta, \xi^{\prime} \otimes \eta^{\prime}\right\rangle=\left\langle\left\langle\xi, \xi^{\prime}\right\rangle \eta, \eta^{\prime}\right\rangle$ for $\xi, \xi^{\prime} \in \mathscr{E}$ and $\eta, \eta^{\prime} \in \mathscr{H}$. In the simple case where $\mathscr{E}$ is the free module $\mathscr{A}^{q}$ (i.e. $E$ is the trivial bundle with fiber $\mathbb{C}^{q}$ ), the connection is given by a matrix $\omega=\omega_{i j}$ of elements of $\Omega^{1}$, with $i, j \in\{1, \ldots, q\}$ and the curvature is the operator in $\mathscr{H}^{q}$ given by the matrix $d \omega+\omega^{2}$, with $\left(d \omega+\omega^{2}\right)_{i k}=d\left(\omega_{i k}\right)+\sum \omega_{i j} \omega_{j k}$. In general if $\theta$ is the curvature, $\theta=\nabla^{2} \in \operatorname{Hom}\left(\mathscr{E}, \mathscr{E} \otimes_{\mathscr{A}} \Omega^{2}\right)$, of the connection $\nabla$, there exists elements $\xi^{i}$ of $\mathscr{E}$, $i \in\{1, \ldots, q\}$ and $\theta_{i j} \in \Omega^{2} ; i, j \in\{1, \ldots, q\}$ such that $\theta(\xi)=\Sigma\left(\xi^{i} \otimes \theta_{i j}\right)\left\langle\xi^{j}, \xi\right\rangle$. The corresponding operator in $\mathscr{E} \otimes_{\mathscr{A}} \mathscr{H}$ is then such that:

$$
\theta(\xi \otimes \eta)=\sum \xi^{i} \otimes \theta_{i j}\left(\left\langle\xi^{j}, \xi>\eta\right) \forall \xi \in \mathscr{E}, \eta \in \mathscr{H} .\right.
$$

The compatibility of the connection $\nabla$ with the metric implies that $\theta$ is a selfadjoint operator in $\mathscr{E} \otimes_{\mathscr{A}} \mathscr{H}:$ If $\mathscr{E}=\mathscr{A}^{q}$, then the connection given by $\omega=\left(\omega_{i j}\right) \in M_{q}\left(\Omega^{1}\right)$ is compatible iff $\omega^{*}=-\omega$ and the curvature $\theta=d \omega+\omega^{2}$ is then selfadjoint since for $\alpha \in \Omega^{1}$ one has $d \alpha^{*}=-(d \alpha)^{*} \in \Omega^{2}$. For the sake of clarity, since we are going to relate our notion of connection with the usual notion we shall use the term $q$-connection for the former and $c$-connection for the latter.

Lemma 11. a) Every q-connection $\nabla: \mathscr{E} \rightarrow \mathscr{E} \otimes \Omega^{1}$ determines uniquely a classical connection $\nabla_{c}$ by composition with the bimodule map $c: \Omega^{1} \rightarrow A^{1}$ of Theorem 3: $\nabla_{c}=(1 \otimes c) \circ \nabla$.

b) Let $\theta$ be the curvature of the q-connection $\nabla$, then the curvature $\theta_{c}$ of $\nabla_{c}$ is the antisymmetric part $A c(\theta)$ of $c(\theta)$.

Proof. a) One has $c(a \alpha b)=a c(\alpha) b$ for $a, b \in \mathscr{A}, \alpha \in \Omega^{1}$, so $(1 \otimes c) \circ \nabla$ is a linear map of $\mathscr{E}=C^{\infty}(M, E)$ to $\mathscr{E} \otimes{ }_{\mathscr{A}} A^{1}=C^{\infty}\left(M, E \otimes T^{*}\right)$ such that $\nabla_{c}(\xi a)=\left(\nabla_{c} \xi\right) a+\xi \otimes d a$ for any $\xi \in \mathscr{E}, a \in \mathscr{A}$.

b) Since the ordinary exterior product of two 1 -forms is the antisymmetric part of their tensor product, the answer follows from Lemma 7.

Corollary 12. The map $\nabla \rightarrow \nabla_{c}$ maps flat $q$-connections to ordinary flat connections on $\mathscr{E}$.

Note that the flatness of the $q$-connection $\nabla$ means as in Theorem 9 that the operator $F_{\nabla}=1 \otimes F-i \nabla$ in the Hilbert space $\mathscr{E} \otimes \mathscr{A} \mathscr{H}$ satisfies $F_{\nabla}^{2}=1$, and hence, in the compatible case, yields an element of a suitable Grassmanian. Here $F_{\nabla}$ is defined by: $F_{\nabla}(\xi \otimes \eta)=\xi \otimes F \eta-i \sum \xi^{j} \otimes \omega_{j} \eta$, with $\nabla \xi=\sum \xi^{j} \otimes \omega_{j} \in \mathscr{E} \otimes \otimes_{\mathscr{A}} \Omega^{1}$. One checks that the right-hand side is independent of any choice. Now by Lemma 7 we can associate to every $q$-connexion a classical tensorial data which is a bit more refined than a classical connexion. Indeed the bimodule $\Omega^{1} / \Omega_{00}^{1}=\sum$ is by Lemma 7 isomorphic to the space of smooth tensors $C^{\infty}\left(M, T^{1} \oplus T^{2}\right)$ which satisfy the equation $d \omega=A \beta$, and the bimodule structure of $\sum$ is given by: $a(\omega, \beta)$ $=(a \omega, d a \otimes \omega+a \beta) ;(\omega, \beta) a=(\omega a, \beta a-\omega \otimes d a)$. By the map $(\omega, \beta) \rightarrow(\omega, \beta-d \omega)$, we can identify $\sum$ with the space of all smooth tensors $C^{\infty}\left(M, T^{1} \oplus S^{2} T^{1}\right)$ with the bimodule structure given by:

$$
a(\omega, \sigma)=(\omega, \sigma) a=\left(a \omega, a \sigma+\frac{1}{2}(d a \otimes \omega+\omega \otimes d a)\right)
$$


$=(a \omega, a \sigma+d a \cdot \omega)$, where $d a \cdot \omega$ is the product in the symmetric algebra. Note in particular that the map $(\omega, \sigma) \rightarrow \omega$ is an $\mathscr{A}$-bimodule map of $\sum$ to $A^{1}$, but that the subspace $\left\{(\omega, \sigma) \in \sum ; \sigma=0\right\}$ is not a submodule of $\sum$.

Lemma 13. 1. The map $\nabla \rightarrow(1 \otimes \tilde{c}) \circ \nabla$ is a surjection of the space of $q$-connections on $\mathscr{E}$ to the space $\Gamma_{\mathscr{E}}$ of maps $\chi: \mathscr{E} \rightarrow \mathscr{E} \otimes \mathscr{A} \sum$ such that $\chi(\xi a)=\chi(\xi) a+\xi \otimes d a \forall \xi \in \mathscr{E}$, $a \in \mathscr{A}$.

2. The map $(\omega, \sigma) \rightarrow \omega$ gives a surjection $\varrho$ of $\Gamma_{\mathscr{E}}$ on the space of classical connections on $E$, and the fibers of $\varrho$ are affine spaces over the vector space $C^{\infty}\left(M\right.$, End $\left.E \otimes S^{2} T^{*}\right)$ of smooth 2-tensors.

Proof. 1. To prove 1. one can assume, as in [3, Proposition 19], that $\mathscr{E}=\mathscr{A}^{n}$, so that a $q$-connection is an element of $M_{n}\left(\Omega^{1}\right)$ and $\Gamma_{\mathscr{E}}=M_{n}(\Sigma)$, thus 1 . follows from Lemma 7.

2. We view $C^{\infty}\left(M, S^{2} T^{*}\right)$ as a submodule $\sum_{0}$ of $\sum$ by the map $\sigma \rightarrow(0, \sigma)$. One has $C^{\infty}\left(M\right.$, End $\left.E \otimes S^{2} T^{*}\right)=\operatorname{Hom}_{\mathscr{A}}\left(\mathscr{E}, \mathscr{E} \otimes \sum_{\mathscr{A}} \sum_{0}\right)$. Thus the exact sequence of bimodules:

$$
0 \rightarrow \sum_{0} \rightarrow \sum \rightarrow A^{1} \rightarrow 0
$$

gives the desired answer.

Theorem 14. Let $M$ be a 4-dimensional $\operatorname{Spin}^{c}$ Riemannian compact manifold, $\mathscr{H}=L^{2}(M, S)$ and $F=D|D|^{-1}$ as above, and $E$ a hermitian vector bundle over $M$, $\mathscr{E}=C^{\infty}(M, E)$.

1. For every compatible q-connection $\nabla$ on $\mathscr{E}$, the curvature $\theta \in \mathscr{L}\left(\mathscr{E} \otimes \otimes_{\mathscr{A}} \mathscr{H}\right)$ belongs to $\mathscr{L}^{2+}$ and the value of the Dixmier trace Trace ${ }_{\omega}\left(\theta^{2}\right)=I(\theta)$, is independent of $\omega$ and defines a gauge invariant positive functional $I$.

2. The restriction of I to each (affine space) fiber of the map $\nabla \rightarrow \nabla_{c}$ is Gaussian (i.e. a quadratic form) and one has:

$$
\operatorname{Inf}_{\nabla_{c}=A} I(\nabla)=\left(16 \pi^{2}\right)^{-1} \mathrm{YM}(A)
$$

where $A$ is a classical connection and YM the classical Yang Mills action.

In fact we shall prove more since we shall identify the Hilbert space of the Gaussian as $L^{2}\left(M\right.$, End $\left.E \otimes S^{2} T^{*}\right)$.

Proof. 1. Follows from the inclusion $\Omega^{2} \subset \mathscr{L}^{2+}$, i.e. Lemma 4, 1) and Theorem 1. The gauge invariance (under the unitary group of End $\left.\mathscr{A}_{\mathscr{A}} \mathscr{E}\right)$ ) follows from the trace property of Trace . $_{\text {. }}$

2. The value of $I(\theta)$ depends only upon the element $\chi$ of $\Gamma$ associated to the $q$-connection $\nabla$. In order to see that and to compute $I(\theta)$ we shall for simplicity assume that $\mathscr{E}=\mathscr{A}^{n}$. Then $\nabla$ is given by a matrix $\left(\alpha_{i j}\right), \alpha_{i j} \in \Omega^{1}$, with $\alpha_{j i}=-\alpha_{i j}^{*} \forall i$, $j \in\{1, \ldots, n\}$. The curvature $\theta$ is given by the matrix $\left(\theta_{i j}\right), \theta=d \alpha+\alpha^{2}$, i.e. $\theta_{i j}=d \alpha_{i j}+\sum_{k} \alpha_{i k} \alpha_{k j}$. Since $\alpha_{i j} \in \Omega^{1}$, one has $\left(d \alpha_{i j}\right)^{*}=d \alpha_{j i}$ and $\theta_{i j}^{*}=\theta_{j i}$. Now the value of $\operatorname{Tr}_{\omega}\left(\theta^{2}\right)$ only depends upon the image of $\theta$ in $\Omega^{2} / \Omega_{0}^{2}$, and the latter only depends upon the image $\tilde{c}\left(\alpha_{i j}\right)$ of $\alpha_{i j}$ in $\Omega^{1} / \Omega_{00}^{1}$, thus our assertion. Now let us write $\tilde{c}\left(\alpha_{i j}\right)$ $=\left(\omega_{i j}, \beta_{i j}\right)$ with $A \beta_{i j}=d \omega_{i j}$ as in Lemma 7. Then the image $c\left(\theta_{j}\right)$ of $\theta_{i j}$ in $\Omega^{2} / \Omega_{0}^{2}$, considered as a tensor of rank 2 , is given by the following formula:

$$
c\left(\theta_{i j}\right)=\beta_{i j}+\sum_{k} \omega_{i k} \omega_{k j}
$$


For each $i j$ the antisymmetric part $A c\left(\theta_{i j}\right)$ is the $i, j$ component of the curvature of the associated classical connection (cf. 11b)). By 132 2., the symmetric part of the tensors $\beta_{i j}$ is any smooth symmetric tensor $t_{i j}$ with $t_{j i}=t_{i j}^{*} \forall i, j$, [where $(\xi \otimes \eta)^{*}$ $=\eta^{*} \otimes \xi^{*}$ for any tensors of rank $1, \xi$ and $\left.\eta\right]$. By Theorem 1, there exists an $O(4)$ invariant inner product on $T^{2} \mathbb{R}^{4}=\Lambda^{2} \mathbb{R}^{4} \oplus S^{2} \mathbb{R}^{4}$ such that, with the above notations:

$$
I(\nabla)=\operatorname{Trace}_{\omega}\left(\theta^{2}\right)=\int_{M}\left\|c\left(\theta_{i j}\right)\right\|^{2} .
$$

Since in this inner product $\Lambda^{2} \mathbb{R}^{4}$ is necessarily orthogonal to $S^{2} \mathbb{R}^{4}$, it follows that, while $I(\nabla)$ obviously depends quadratically on the symmetric part of $\beta_{i j}$, its minimum over each fiber of $\nabla \rightarrow \nabla_{c}$ is reached when the symmetric part of each tensor $c\left(\theta_{i j}\right)$ is set equal to 0 . But then the value of $I(V)$ is, up to a numerical factor, the standard Yang-Mills action.

\section{References}

1. Adler, M.: On a trace functional for formal pseudodifferential operators and the symplectic structure of the Korteweg of Wries type equations. Invent. Math. 50, 219-248 (1979)

2. Alvarez-Gaumé, L., Gomez, C.: New methods in string theory. Preprint CERN (1987)

3. Connes, A.: Non-commutative differential geometry. Publ. Math. IHES 62, 257-360 (1985)

4. Connes, A., Rieffel, M.: Yang-Mills for non-commutative two tori. Contemp. Math. 62, 237-266 (1987)

5. Connes, A.: Compact metric spaces, Fredholm modules and Hyperfiniteness. Preprint (1987). J. Ergodic Theory (to appear)

6. Dixmier, J.: Existence de traces non normales. C.R. Acad. Sci. Paris 262, 1107-1108 (1966)

7. Gohberg, I., Krein, M.G.: Introduction to the theory of non-selfadjoint operators. Moscow (1985)

8. Manin, Y.I.: Algebraic aspects of non-linear differential equations. J. Sov. Math. 11, 1-122 (1979)

9. Pressley, A., Segal, G.: Loop groups. Oxford: Oxford Science 1986

10. Segal, G., Wilson, G.: Loop groups and equations of KdV type. Publ. Math. IHES 61, 5-65 (1985)

11. Weyl, H.: Classical groups. Princeton, NJ: Princeton University Press

12. Witten, E.: Non-commutative geometry and string field theory

13. Wodzicki, M.: Local invariants of spectral assymmetry. Invent. Math. 75, 143-178 (1984)

14. Guillemin, V.W.: A new proof of Weyl's formula on the asymptotic distribution of eigenvalues. Adv. Math. 55, 131-160 (1985)

Communicated by A. Jaffe

Received January 4, 1988; in revised form February 9, 1988 
